\title{
4
}

\section{FREQUENCY DISTRIBUTION OF THE VALUES OF THE CORRELATION COEFFICIENT IN SAMPLES FROM AN INDEFINITELY LARGE POPULATION.}

\author{
BY R. A. FISHER.
}

1. My attention was drawn to the problem of the frequency distribution of the correlation coefficient by an article published by Mr H. E. Soper* in 1913. Seeing that the problem might be attacked by means of geometrical ideas, which $I$ had previously found helpful in the consideration of samples, I have examined the two articles by "Studentf," upon which Mr Soper's more elaborate work was based, with a view to checking and verifying the conclusions there attained.

"Student," if I do not mistake his intention, desiring primarily to obtain a just estimate of the accuracy to be ascribed to the mean of a small sample, found it necessary to allow for the fact that the mean square error of such a sample is not generally equal to the standard deviation of the normal population from which it is drawn. He was led, in fact, to study the frequency distribution of the mean square error. He calculated algebraically the first four moment: of this frequency curve, both about the zero point, and about its mean, observed a simple law to connect the successive moments, and discovered a frequency curve, which fitted his moments, and gave the required law.

Thus if $x_{1}, x_{2}, \ldots x_{n}$ are the members of a sample,

and

$$
n \bar{x}=x_{1}+x_{2}+\ldots+x_{n},
$$

$$
n \mu^{2}=\left(x_{1}-\bar{x}\right)^{2}+\left(x_{2}-\ddot{x}\right)^{2}+\ldots+\left(x_{n}-\bar{x}\right)^{2},
$$

the frequency with which the mean square error lies in the range $d \mu$ is proportional to

$$
\mu^{n-2} e^{-\frac{n \mu^{2}}{2 \sigma^{2}}} d \mu
$$

This result, although arrived at by empirical methods, was established almost beyond reasonable doubt in the first of "Student's" papers. It is, however, of interest to notice that the form establishes itself instantly, when the distribution of the sample is viewed geometrically.

\footnotetext{
* Biometrika, Vol, rx. p. 91. † Ibid. Vol. vi. pp. 1 and 302,
} 


\section{Distribution of the Correlation Coefficients of Samples}

In the second of these two papere the more difficult problem of the frequency distribution of the correlation coefficient is attempted. For samples of 2 the frequency distribution between the only two possible values -1 and +1 was determined by Sheppard's theorem to be in the ratio $\frac{\pi}{2}+\sin ^{-1} \rho: \frac{\pi}{2}-\sin ^{-1} \rho$, where $\rho$ is the correlation of the population. Besides this theoretical result, "Student" appeals only to experimental data. From these he derives an empirical form for the distribution when $\rho=0$, and makes several valuable suggestions. It has been the greatest pleasure and interest to myself to observe with what accuracy "Student's" insight has led him to the right conclusions. The form when $\rho=0$ is absolutely correct, and as a further instance I may quote the remark* "I have dealt with the cases of samples of 2 at some length, because it is possible that this limiting value of the distribution, with its mean of $\frac{2}{\pi} \sin ^{-1} \rho$ and its second moment coefficient of $1-\left(\frac{2}{\pi} \sin ^{-1} \rho\right)^{2}$, may furnish a clue to the distribution when $n$ is greater than 2." As a matter of fact it is just these quantities with which we shall be concerned.

To Mr Soper's laborious and intricate paper I cannot hope to do justice. I have been able to establish the substantial accuracy and value of his approximations. It is one of the advantages of approaching a problem from opposite standpoints that $\mathrm{Mr}$ Soper's forms are most accurate for those larger values of $n$, where the exact formulae become most complicated.

2. The problem of the frequency distribution of the correlation coefficient $r$, lerived from a sample of $n$ pairs, taken at random from an infinite population, may be solved, when that population can be represented by a normal surface, with the aid of certain very general conceptions derived from the geometry of $n$ dimensional space. In this paper the general form will first be demonstrated, and for a few important cases some of the successive moments will be derived. Incidentally it will be of interest to compare the exact form with $\mathrm{Mr}$ Soper's approximation, and with reference to the experimental data supplied by "Student."

If the frequency distribution of the population be specified by the form

$$
\left.d f=\frac{1}{2 \pi \sigma_{1} \sigma_{2} \sqrt{1-\rho^{2}}} e^{-\frac{1}{1-\rho^{2}}\left\{\frac{\left(x-m_{1}\right)^{2}}{2 \sigma_{1}^{2}}-\frac{2 \rho\left(x-m_{1}\right)\left(y-m_{2}\right)}{2 \sigma_{1} \sigma_{2}}+\frac{\left(y-m_{2}\right)^{2}}{2 \sigma_{2}^{2}}\right.}\right\} d x d y,
$$

where $d f$ is the chance that any observation should fall into the range $d x d y$, then the chance that $n$ pairs should fall within their specified elemeuts is

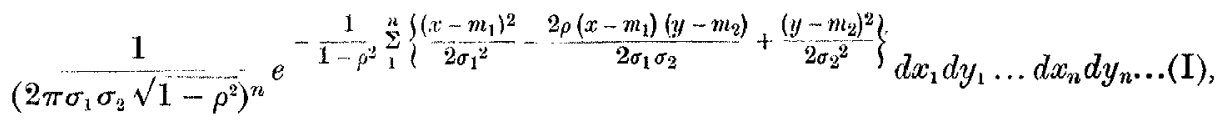

and this we interpret as a simple density distribution in $2 n$ dimensions.

* Biometrika, Vol. vi. p. 304. 
For the variables $x$ and $y$ it is now necessary to substitute the statistical derivatives determined by the equations

$$
\begin{array}{rlrl}
n \bar{x} & =\sum_{1}^{n}(x), & n \bar{y} & =\sum_{1}^{n}(y), \\
n \mu_{1}{ }^{2} & =\sum_{1}^{n}(x-\bar{x})^{2}, \quad n \mu_{2}{ }^{2}=\sum_{1}^{n}(y-\bar{y})^{2}, \\
n r \mu_{1} \mu_{2} & =\sum_{1}^{n}(x-\bar{x})(y-\bar{y}),
\end{array}
$$

and it is evident that the only difficulty lies in the expression of an element of volume in $2 n$ dimensional space in terms of these derivatives.

The five quantities above defined have, in fact, an exceedingly beautiful interpretation in generalised space, which we may now examine.

3. Considering first the space of $n$ dimensions in which the variations of $x$ are represented, the mean and mean square error of $n$ observations are determined by the relations of $P$, the point representing the $n$ observations, to the line

$$
x_{1}=x_{2}=x_{3}=\ldots=x_{n},
$$

for the perpendicular $P_{\mathrm{d}} / L$ drawn from $P$ upon this line will lie in the regriou

$$
x_{1}+x_{2}+\ldots+x_{n}=n i c
$$

and will neet it at the point $M$, where

$$
x_{1}=\bar{x}, \quad x_{2}=\bar{r}, \ldots x_{n}=\bar{x} ;
$$

further, since,

$$
P M^{2}=\left(a_{1}-\bar{a}\right)^{2}+\left(x_{n}-\bar{x}\right)^{2}+\ldots+\left(x_{n}-i\right)^{3},
$$

the length of $P M$ is $\mu_{1} \sqrt{ } n$.

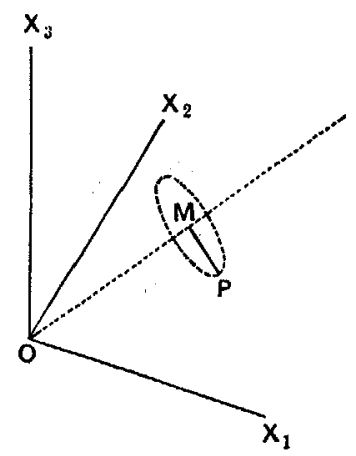

An element of volume in this $n$ dimensional space may now without difficulty be specified in terms of $\bar{x}$ and $\mu_{1}$; for, given $\bar{x}$ and $\mu_{1}, P$ must lie on a sphere in $n-1$ dimensions, lying at right angles to the line $O M$, and the element of volume is

$$
C \mu_{1}{ }^{n-2} d \mu_{1} d \bar{x}
$$

where $C$ is some constant, which need not be determined. 
The point in $2 n$ dimensional space which is represented by the $n$ pairs of observations must be such that its projection on the $n$ dimensional space, in which $x$ is represented, lies upon a certain sphere of radius $\mu_{1} \sqrt{ } n$, and on the space in which $y$ is represented, upon another sphere of radius $\mu_{2} \sqrt{ } n$, and now, when we come to the interpretation of $r$, we must observe that to each point on the first sphere there corresponds a certain point on the second sphere, to which it bears the relation

$$
\frac{x_{1}-\bar{x}}{y_{1}-\bar{y}}=\frac{x_{2}-\bar{x}}{y_{2}-\bar{y}}=\ldots=\frac{x_{n}-\bar{x}}{y_{n}-\bar{y}}
$$

In general this relation does not hold for the $n$ pairs of observations, and the two projections will not fall at corresponding points on the two spheres. If now one of the spheres be turned round so as to occupy the same space as the other, and so that the lines upon which $x_{1}$ and $y_{1}$, and the other pairs of coordinates, are measured, coincide, then corresponding points will lie on the same radii, and the correlation coefficient $r$ measures the cosine of the angle between the radii to the two points specified by the observations.

* Taking one of the projections as fixed at any point on the sphere of radius. $\mu_{2}$ the region for which $r$ lies in the range $d r$, is a zone, on the other sphere in $n-1$ dimensions, of radius $\mu_{1} \sqrt{n} \sqrt{1-r^{2}}$, and of width $\mu_{1} \sqrt{n} d r / \sqrt{1-r^{2}}$, and therefore having a volume proportional to $\mu_{1}^{n-2}\left(1-r^{2}\right)^{\frac{n-4}{2}} d r$.

4. We may now turn to the direst simplification of the expression (I), at each stage discarding any factors which do not involve $r$.

$$
e^{-\frac{1}{1-\rho^{2}} \sum_{1}^{2}\left\{\frac{\left(x-m_{1}\right)^{2}}{2 \sigma_{1}^{2}}-\frac{2 \rho\left(x-m_{1}\right)\left(y-m_{2}\right)}{2 \sigma_{1} \sigma_{2}}+\frac{\left(y-m_{2}\right)^{2}}{2 \sigma_{2}^{2}}\right\}} d x_{1} d y_{1} d x_{2} d y_{2} \ldots d x_{n} d y_{n}
$$

may be reduced to

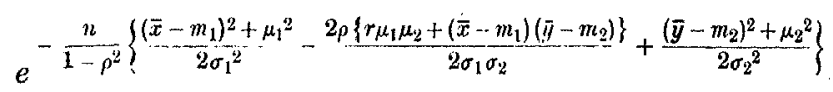

$$
\begin{aligned}
& d \bar{x} d \bar{y} \mu_{2}^{n-2} d \mu_{1} \mu_{2}^{n-2} d \mu_{2}\left(1-r^{2}\right)^{\frac{n-4}{2}} d r,
\end{aligned}
$$

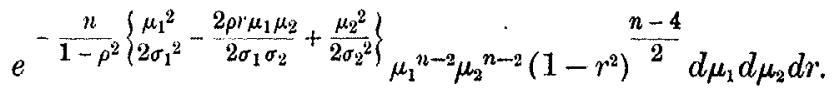

or to

In order to integrate this expression from 0 to $\infty$, with respect to $\mu_{1}$ and $\mu_{2}$, let

and we have

$$
\zeta=\frac{\mu_{1} \mu_{2}}{\sigma_{1} \sigma_{2}}, \quad e^{z}=\frac{\mu_{1} \sigma_{2}}{\mu_{2} \sigma_{1}},
$$

$$
\begin{aligned}
\int_{-\infty}^{\infty} d z \int_{0}^{\infty} \zeta^{n-2} d \zeta \cdot e^{-\frac{n}{1-\rho^{2}}(\cosh z-\rho r) \zeta} \cdot\left(1-r^{2}\right)^{\frac{n-4}{2}} d r, \\
\text { or } \\
\qquad \int_{0}^{\infty} \frac{d z}{(\cosh z-\rho r)^{n-1}} \cdot\left(1-r^{2}\right)^{\frac{n-4}{2}} d r
\end{aligned}
$$


which, on substituting $\cos \theta$ for - $\rho r$, may be expressed in terms of a Legendre function in the form

$$
(i \operatorname{cosec} \theta)^{n-1} Q_{n-2}(i \cot \theta) \cdot\left(1-r^{2}\right)^{\frac{n-4}{2}} d r
$$

Again

$$
\int_{0}^{\infty} \frac{d z}{\cosh z+\cos \theta}=\frac{\theta}{\sin \theta}
$$

so that

$$
\int_{0}^{\infty} \frac{d z}{(\cosh z+\cos \theta)^{n-1}}=\frac{1}{n-2}\left(\frac{\partial}{\sin \theta \partial \theta}\right)^{n \sim 2} \frac{\theta}{\sin \theta},
$$

and since this is a function of $\rho r$ only, we may express the frequency distribution by the convenient expression

$$
\left(1-r^{2}\right)^{\frac{n-4}{2}} \frac{\partial^{n-2}}{\partial r^{n-2}}\left(\frac{\theta}{\sin \theta}\right) d r
$$

Professor Pearson has shown that this last result can be nbtained directly from Sheppard's theorem* that

$$
\frac{1}{2 \pi \Sigma_{1} \Sigma_{2} \sqrt{1-R^{2}}} \int_{0}^{\infty} \int_{0}^{\infty} e^{-\frac{1}{2\left(1-\Sigma^{2}\right)}\left(\frac{\mu_{1}^{2}}{\Sigma_{1}^{2}}-\frac{2 R \mu_{1} \mu_{2}}{\Sigma_{1} \Sigma_{2}}+\frac{\mu_{2}^{2}}{\Sigma_{2}^{2}}\right)} d \mu_{1} d \mu_{2}=\frac{\cos ^{-1}(-R)}{2 \pi}
$$

making the substitutions

which give

$$
\begin{aligned}
\frac{1}{\left(1-R^{2}\right) \Sigma_{1}^{2}} & =\frac{n}{\left(1-\rho^{2}\right) \sigma_{1}^{2}}, \\
\frac{1}{\left(1-R^{2}\right) \Sigma_{2}^{2}} & =\frac{n}{\left(1-\rho^{2}\right) \sigma_{2}^{2}}, \\
\frac{R}{\left(1-R^{2}\right) \Sigma_{1} \Sigma_{2}} & =\frac{n r \rho}{\left(1-\rho^{2}\right) \sigma_{1} \sigma_{2}}, \\
R & =\rho r \\
\cos ^{-1}(-R) & =\theta,
\end{aligned}
$$

and

we obtain

$$
\frac{n}{\sigma_{1} \sigma_{2}\left(1-\rho^{2}\right)} \int_{0}^{\infty} \int_{0}^{\infty} e^{-\frac{n}{2\left(1-\rho^{2}\right)}\left(\frac{\mu_{1}^{2}}{\sigma_{1}^{2}}-\frac{2 \rho r \mu_{1} \mu_{2}}{\sigma_{1} \sigma_{2}}+\frac{\mu_{2}^{2}}{\sigma_{2}^{2}}\right)} d \mu_{1} d \mu_{2}=\frac{\theta}{\sin \theta}
$$

and hence differentiating $(n-2)$ times with respect to $r$, the required expression is obtained.

5. The form which we have now obtained may be applied without difficulty to all small even values of $n$, and in such cases is peculiarly suitable for the calculation of moments.

When $n=2$ the ordinate of the curve, with abscissa $r$, is

$$
\frac{\theta}{\left(1-r^{2}\right) \sin \theta}
$$

which becomes hyperbolic in the neighbourhoods of -1 and +1 . The value

* Phil. Trans. Vol. 192, A, p. 141. 


\section{Distribution of the Correlation Coefficients of Samples}

of $r$ is, therefore, as we know, either -1 or +1 , and the proportion, in which these occur, depends upon $\rho$. The ratio of the infinite areas included with the asymptotes of the above curve is

$$
\frac{\cos ^{-1} \rho}{\cos ^{-1}(-\rho)}
$$

so that the mean value of a number of observations is $\frac{\sin ^{-1} \rho}{\frac{\pi}{2}}$.

When $n=4$ there is still no approach to normality, the curve takes the form

$$
\frac{1}{\sin ^{3} \theta}\left(\theta-3 \cot \theta+3 \theta \cot ^{2} \theta\right)
$$

which, when $r$ is positive, increases regularly from its value of $\frac{4}{15}$ when $\theta=0$, to infinity, to which it approaches as $\theta$ approaches $\pi$. Unless $\rho$ is actually equal to 1 , in which case $r$ is also 1 of necessity, the curve has finite ordinates at both extremes. For calculating the number of values which should fall within any given range, the integral, $\frac{1}{\sin ^{2} \theta}(1-\theta \cot \theta)$, may be directly tabulated, as has been done in forming the accompanying table of "Student's" observations, and the corresponding expectations. The values given by $\mathrm{Mr}$ Soper's formula are apposed for comparison.

\begin{tabular}{|c|c|c|c|c|c|c|c|}
\hline$r$ & $\begin{array}{c}\text { Calculated } \\
\text { frequency } \\
m\end{array}$ & Observed & $\begin{array}{c}\text { Difference } \\
e^{\prime}\end{array}$ & $\frac{62}{m}$ & $\begin{array}{c}\text { H.E.Soper's } \\
\text { approxi- } \\
\text { mation }\end{array}$ & $\begin{array}{c}\text { Diff erence } \\
e\end{array}$ & $\frac{e^{-2}}{m}$ \\
\hline$\cdot 905-1$ & $202 \cdot 1$ & 175.5 & -15.0 & $\cdot 69$ & $230 \cdot 3$ & $-17 \cdot 2$ & 90 \\
\hline$\cdot 805-905$ & $124 \cdot 9$ & $136 \cdot 5$ & -100 & Oi & $98 \cdot 9$ & & \\
\hline $705-805$ & $88 \cdot 7$ & $\begin{array}{l}84 \\
66\end{array}$ & $-3 \cdot 8$ & .09 & $\begin{array}{l}72 \cdot 1 \\
57 \cdot 6\end{array}$ & $+20 \cdot 3$ & $3 \cdot 18$ \\
\hline $\begin{array}{l}\cdot 605- \\
.505-\end{array}$ & $\begin{array}{l}65 \cdot 1 \\
49 \cdot 9\end{array}$ & $\begin{array}{l}66 \\
55\end{array}$ & & & 48.0 & $+11 \cdot 8$ & \\
\hline$\cdot 405-$ & $37 \cdot 8$ & 45 & $+12 \cdot 3$ & {$[\cdot 73$} & $40 \cdot 2$ & +118 & 1.58 \\
\hline $\begin{array}{l}305- \\
.205-\end{array}$ & $\begin{array}{l}30 \cdot 6 \\
24 \cdot 8\end{array}$ & $\begin{array}{l}24 \cdot 5 \\
24 \cdot 5\end{array}$ & -64 & $\cdot 74$ & $\begin{array}{l}34 \cdot 3 \\
29 \cdot 7\end{array}$ & $-15 \cdot 0$ & 3.52 \\
\hline$\cdot 105-$ & $20 \cdot 5$ & 19 & $-11 \cdot 6$ & $3 \cdot 58$ & 25.6 & $-21 \cdot 6$ & $9 \cdot 80$ \\
\hline $\begin{array}{l}\cdot 005- \\
1.905-\end{array}$ & $17 \cdot 1$ & $\begin{array}{r}7 \\
22\end{array}$ & & & 18.8 & 0 & \\
\hline $1.805-$ & $\begin{array}{l}14 \cdot 5 \\
12 \cdot 4\end{array}$ & 12 & $+7 \cdot 1$ & $1 \cdot 87$ & 16.0 & -8 & 0.02 \\
\hline $1 \cdot 705$ & $10 \cdot 7$ & 13 & $-4 \cdot 0$ & $\cdot 80$ & 13.5 & $-8 \cdot 7$ & 3.06 \\
\hline $1 \cdot 605-$ & $9 \cdot 3$ & 3 & -40 & & $11 \cdot 2$ & o & \\
\hline $\begin{array}{l}\overline{1} \cdot 505-- \\
\overline{1} \cdot 405-\end{array}$ & $\begin{array}{l}8 \cdot 1 \\
7 \cdot 2\end{array}$ & $\begin{array}{l}12 \\
16\end{array}$ & $+12 \cdot 7$ & $10 \cdot 54$ & $\begin{array}{l}9 \cdot 0 \\
6.9\end{array}$ & $+12 \cdot 1$ & $9 \cdot 21$ \\
\hline$\frac{1}{1} \cdot 305-305-$ & $\begin{array}{l}72 \\
6 \cdot 3\end{array}$ & 7 & 5.1 & 0.19 & $5 \cdot 1$ & $+8 \cdot 6$ & $8 \cdot 80$ \\
\hline $1 \cdot 205$ & $5 \cdot 6$ & 10 & +51 & 219 & $3 \cdot 3$ & +00 & 000 \\
\hline$\overline{\overline{1}} \cdot 105-\overline{\mathrm{I}} \cdot 105$ & $\begin{array}{l}5 \cdot 1 \\
4 \cdot 3\end{array}$ & $\begin{array}{l}4 \\
9\end{array}$ & +3.6 & $1 \cdot 38$ & $\begin{array}{r}1 \cdot 9 \\
\cdot 6\end{array}$ & +10.5 & $44 \cdot 10$ \\
\hline - & -- & 745 & - & $23 \cdot 61$ & - & - & $84 \cdot 17$ \\
\hline & & & & & & & \\
\hline
\end{tabular}

Table for comparison with p. 114, Biometrika, Vol. IX. 
6. The direct process of integration by parts applied to such expressions as

$$
\int_{-1}^{+1}\left(1-r^{2}\right)^{\frac{n-4}{2}} \frac{\partial^{n-1}}{\partial r^{n-1}} \frac{\theta^{2}}{2} d r \text { and } \int_{-1}^{+1}\left(1-r^{2}\right)^{\frac{n-4}{2}} r \frac{\partial^{n-1}}{\partial r^{n-1}} \frac{\theta^{2}}{2} d r
$$

when $n$ is even, merely introduces the sums and differences of the terms $\frac{\partial^{p}}{\partial r^{p}} \frac{\theta^{2}}{2}$ at the extremes, where $r$ is -1 or +1 , with coefficients which are, in any particular case, easily calculable.

Thus, $n$ being 6 ,

$$
\begin{aligned}
& \int_{-1}^{+1}\left(1-r^{2}\right) \frac{\partial^{5}}{\partial r^{5}} \frac{\theta^{2}}{2} d r=\left[\left(1-r^{2}\right) \frac{\partial^{4}}{\partial r^{4}} \frac{\theta^{2}}{2}\right]_{-1}^{+1}+\left[2 r \frac{\partial^{3}}{\partial r^{3}} \frac{\theta^{2}}{2}\right]_{-1}^{+1}-\left[2 \frac{\partial^{2}}{\partial r^{2}} \frac{\theta^{2}}{2}\right]_{-1}^{+1} \\
& =2 \times \text { the sum of the extreme values of } \frac{\rho^{3}}{\sin ^{3} \theta}\left(\theta-3 \cot \theta+3 \theta \cot ^{2} \theta\right) \\
& -2 \times \text { the difference of the extreme values of } \frac{\rho^{2}}{\sin ^{2} \theta}(1-\theta \cot \theta)
\end{aligned}
$$

If $\rho=\sin \alpha$, so that the extreme values of $\theta$ are $\frac{\pi}{2}-\alpha$ and $\frac{\pi}{2}+\alpha$, the sums and differences may readily be expressed in terms of $\alpha$, and the first few may here be tabulated: the table has been carried back as far as is necessary for the calculation of the fourth moment.

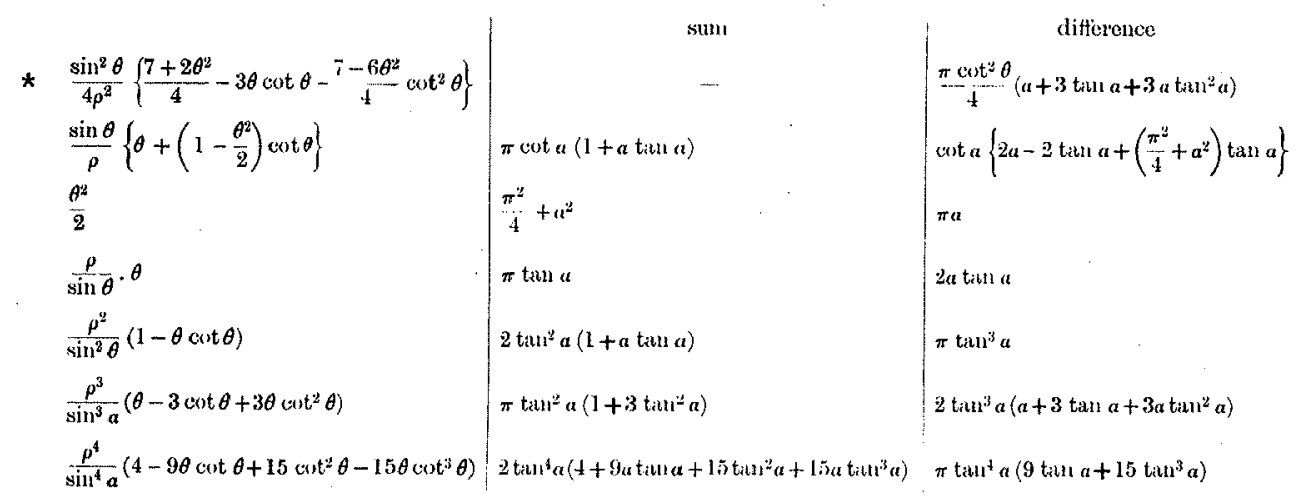

There are here two natural series, which appear alternately as sums and differences; the simpler, which may be expressed in the form

$$
\frac{\pi}{2} \sin ^{p} \alpha\left(\begin{array}{c}
\partial \\
\cos \alpha \partial \alpha
\end{array}\right)^{p} \alpha,
$$




\section{Distribution of the Correlation Coefficients of Samples}

is essentially a series of Legendre functions of the first kind; and may be expressed as

$$
\frac{\pi}{2} \cdot \tan ^{p} \alpha \frac{\mid p-1}{i^{p-1}} P_{p-1}(i \tan \alpha) ;
$$

and it is these only which occur in the evaluation of the even moments.

7. It is, however, desirable to obtain general expressions for these integrals in terms of $n$ and $\rho$, and to evaluate them when $n$ is odd.

For this purpose let us introduce a quantity $\phi$, such that

$$
\cos \phi=\cos \theta-k,
$$

then, when $k$ is sufficiently small, we may expand $\phi^{2}$ by Taylor's theorem, so that

$$
\frac{\phi^{2}}{2}=\frac{\theta^{2}}{2}+k \frac{\partial}{\sin \theta \partial \theta} \frac{\theta^{2}}{2}+\frac{k^{2}}{[2}\left(\frac{\partial}{\sin \theta \partial \theta}\right)^{2} \frac{\theta^{2}}{2}+\ldots
$$

Now let

$$
k=\rho h \sqrt{1-r^{2}} \text {, }
$$

then

$$
\frac{\phi^{2}}{2}=\frac{\theta^{2}}{2}+\rho h \sqrt{1}-\bar{r}^{2} \frac{\partial}{\sin \theta \partial \theta} \frac{\theta^{2}}{2}+\rho^{2} h^{2}\left(1-r^{2}\right)\left(\left.\frac{\partial}{2}\right|^{2}\right)^{2} \frac{\theta^{2}}{2}+\ldots,
$$

and differentiating twice with respect to $h$

$$
\rho^{2}\left(1-r^{2}\right)\left(\frac{\partial}{\sin \phi \partial \phi}\right)^{2} \frac{\phi^{2}}{2}=\rho^{2}\left(1-r^{2}\right)\left(\begin{array}{c}
\partial \\
\sin \theta \partial \theta
\end{array}\right)^{2} \frac{\theta^{2}}{2}+h \rho^{3}\left(1-r^{2}\right)^{3}\left(\frac{\partial}{\sin \theta \partial \theta}\right)^{3} \frac{\theta^{2}}{2}+\ldots
$$

whence, dividing by $\left(1-r^{2}\right)^{\frac{3}{3}}$, we obtain

$$
\underset{\sqrt{ } 1-r^{2}}{\rho^{2}}\left(\begin{array}{c}
\partial \\
\sin \phi \partial \phi
\end{array}\right)^{2} \frac{\phi^{2}}{2}=\frac{\rho^{2}}{\left(1-r^{2}\right)^{\frac{1}{2}}}\left(\frac{\partial}{\sin \theta \partial \theta}\right)^{2} \frac{\theta^{2}}{2}+h \rho^{3}\left(\frac{\partial}{\sin \theta \partial \theta}\right)^{3} \frac{\theta^{2}}{2}
$$

$$
+\frac{\rho^{4} h^{2}}{\mid \underline{2}}\left(1-r^{2}\right)^{\frac{1}{2}}\left(\frac{\partial}{\sin \theta \partial \theta}\right)^{4} \frac{\theta^{2}}{2}+\ldots
$$

so that

$$
\int_{-1}^{+1} r^{p}\left(1-r^{2}\right)^{\frac{n-4}{2}} \frac{\partial^{n-1}}{\partial r^{n-1}} \frac{\theta^{2}}{2} d r
$$

may be obtained by multiplying by $n-3$ the coefficient of $h^{n-3}$ in

$$
\rho^{2} \int_{-1}^{+1} \frac{r^{p} d r}{\sqrt{1-r^{2}}} \cdot \frac{1-\phi \cot \phi}{\sin ^{2} \phi}
$$

when $\cos \phi=\cos \theta-\rho h \sqrt{1-r^{2}}=-\rho\left(r+h \sqrt{1-r^{2}}\right)$.

Our object might equally be achieved by the evaluation of the integral

$$
\rho \int_{-1}^{+1} \frac{r^{p} d r}{1-r^{2}}\left(\frac{\phi}{\sin \phi}-\frac{\theta}{\sin \theta}\right)
$$

The quautity $\phi$ is determined by the equation

$$
\begin{aligned}
& \cos \phi=\cos \theta-\rho h \sqrt{1-r^{2}}, \\
& \cos \phi=-\rho\left(r+h \sqrt{1-r^{2}}\right) .
\end{aligned}
$$


If now

$$
\begin{aligned}
& r=\sin \beta, \\
& h=\tan { }^{2},
\end{aligned}
$$

then

$$
\cos \theta=-\rho \sin \beta \text {, }
$$

$$
\cos \phi=-\rho \sqrt{1+h^{2}} \sin (\beta+\epsilon)=-\rho \sqrt{1+h^{3}} \sin \beta^{\prime},
$$

and as $r$ passes from -1 to +1 ,

$$
\beta \text { passes from }-\frac{\pi}{2} \text { to }+\frac{\pi}{2} \text {, }
$$

$\theta$ from $\frac{\pi}{2}-\alpha$ to $\frac{\pi}{2}+\alpha$,

$\beta^{\prime}$ frem $-\frac{\pi}{2}+\epsilon$ to $\frac{\pi}{2}$ and thence to $\frac{\pi}{2}+\epsilon$,

and $\phi$ from $\frac{\pi}{2}-\alpha$ to $\frac{\pi}{2}+\alpha^{\prime}$ and thence back to $\frac{\pi}{2}+\alpha$,

where $\sin \alpha^{\prime}=\rho \sqrt{1+h^{2}}, \phi$ oscillates in the same manner as $\theta$, with a somewhat greater amplitude, and slightly in advance in respect of phase.

The expression

$$
\rho^{2} \int_{-1}^{+1} \frac{1-\phi \cot \phi}{\sin ^{2} \phi} \frac{d r}{\sqrt{1-r^{2}}}
$$

may now be reduced to

$$
\begin{aligned}
& \rho^{2} \int_{-\frac{\pi}{2}}^{+\frac{\pi}{2}} \frac{1-\phi \cot \phi}{\sin ^{2} \phi} d \beta=\rho^{2} \int_{-\frac{\pi}{2}+\cdot}^{\frac{\pi}{2}+\cdot}\left(\frac{1}{1-\sin ^{2} \alpha^{\prime} \sin ^{2} \beta^{\prime}}+\frac{\phi \sin \alpha^{\prime} \sin \beta^{\prime}}{\left(1-\sin ^{2} \alpha^{\prime} \sin ^{2} \beta^{\prime}\right)^{\frac{3}{2}}}\right) d \beta^{\prime} \\
& =\rho^{2} \int_{-\frac{\pi}{2}}^{+\frac{\pi}{2}} \frac{d \beta^{\prime}}{1-\sin ^{2} \alpha^{\prime} \sin ^{2} \beta^{\prime}}+\pi \rho^{2} \int_{+\frac{\pi}{2}}^{+\frac{\pi}{2}+\rho} \frac{\sin \alpha^{\prime} \sin \beta^{\prime} d \beta^{\prime}}{\left(1-\sin ^{2} \alpha^{\prime} \sin ^{2} \beta^{\prime}\right)^{\frac{1}{2}}} \\
& +\rho^{2} \int_{-\frac{\pi}{2}}^{\frac{\pi}{2}} \frac{(\phi) \sin \alpha^{\prime} \sin \beta^{\prime} d \beta^{\prime}}{\left(1-\sin ^{3} \alpha^{\prime} \sin ^{2} \beta^{\prime}\right)^{\frac{3}{2}}} \\
& =\frac{\rho^{2} \pi}{\cos \alpha^{\prime}}+\frac{\pi \rho^{2} \sin \alpha^{\prime}}{\cos ^{2} \alpha^{\prime}}\left(\frac{\sin \epsilon}{\cos \alpha}\right)+\frac{\pi \rho^{2}}{\cos ^{2} \alpha^{\prime}}\left(1-\cos \alpha^{\prime}\right) \\
& =\frac{\rho^{2} \pi}{\cos ^{2} \alpha^{\prime}}\left(1+\frac{\sin \alpha \tan \epsilon}{\cos \alpha}\right) \text {, } \\
& \cos ^{2} \alpha^{\prime}=1-\rho^{2}\left(1+h^{2}\right)=\cos ^{2} \alpha-\sin ^{2} \alpha \tan ^{2} \epsilon, \\
& \rho^{2} \int_{-1}^{+1} \frac{1-\phi \cot \phi}{\sin ^{2} \phi} \frac{d r}{\sqrt{1-r^{2}}}=\frac{\pi \tan ^{2} \alpha}{1-h \tan \alpha} .
\end{aligned}
$$

From this evaluation we deduce the general form

$$
\int_{-1}^{+1}\left(1-r^{2}\right)^{\frac{n-4}{2}} \frac{\partial^{n-1}}{\partial r^{n-1}} \frac{\theta^{2}}{2} d r=\underline{n-3 \pi \tan ^{n-1} \alpha}
$$




\section{Distribution of the Correlation Coefficients of Samples}

The absolute frequency $d f$, with which $r$ falls in the range $d r$, is therefore

$$
\frac{\left(1-\rho^{2}\right)^{\frac{n-1}{2}}}{\pi n-3}\left(1-r^{2}\right)^{\frac{n-4}{2}}\left(\frac{\partial}{\sin \theta \partial \theta}\right)^{n-2} \frac{\theta}{\sin \theta} d r \text {. }
$$

8. I do not see how to integrate the other expressions of the type

$$
\rho^{2} \int_{-1}^{+1} \frac{1-\phi \cot \phi}{\sin ^{2} \phi} \frac{r^{p} d r}{\sqrt{1-r^{2}}}
$$

although a form could probably be obtained when $p$ is even. The general expression for the second moment may, however, be deduced by means of a reduction formula.

By a process of integration by parts it appears that, if we write

$$
\int_{-1}^{+1}\left(1-r^{2}\right)^{\frac{n-4}{2}} r^{p} \frac{\partial^{n-1}}{\partial r^{n-1}} \frac{\theta^{2}}{2} d r=I_{n, p},
$$

then

$$
I_{n+2.2}=I_{n+2.0}+n I_{n .0}-n(n-1) I_{n .2}
$$

and since

$$
I_{4.2}=2 \pi\left(\frac{\tan ^{3} \alpha}{2}-\tan \alpha+\alpha\right) \text {, }
$$

we may obtain successively

$$
\begin{aligned}
& I_{6.2}=24 \pi\left(\frac{\tan ^{5} \alpha}{4}-\frac{\tan ^{3} \alpha}{3}+\tan \alpha-\alpha\right), \\
& I_{8.2}=720 \pi\left(\frac{\tan ^{7} \alpha}{6}-\frac{\tan ^{5} \alpha}{5}+\frac{\tan ^{3} \alpha}{3}-\tan \alpha+\alpha\right),
\end{aligned}
$$

and so on, yielding, when $n$ is even, the expression

$$
I_{n .2}=I_{n .0}-\pi \mid n-2 \int_{0}^{\alpha} \tan ^{n-2} x d x,
$$

a form which may well hold when $n$ is odd.

The above expressions are useful in tabulating the numerical values of the second moment, $\bar{r}^{2}+\sigma^{2}$, of the unit curve, which may easily be calculated in succession for different values of $n$, when $\tan ^{2} \alpha$ is taken to have some simple value.

9. Before leaving this aspect of the subject it is worth while to give a more detailed examination of the mean of the frequency curves of $r$ when $n=4$.

Two formulae are arrived at by Mr Soper, which are equivalent approximations of the second degree
I. $\quad \bar{r}=\rho\left[1-\frac{1-\rho^{2}}{2 n}\left\{1+\frac{3}{4 n}\left(1+3 \rho^{2}\right)\right\}\right]=\rho\left[1-\frac{1-\rho^{2}}{8}\left\{1+\frac{3}{16}\left(1+3 \rho^{2}\right)\right\}\right]$,
II. $\bar{r}=\rho\left[1-\frac{1-\rho^{2}}{2(n-1)}\left\{1-\frac{1}{4(n-1)}\left(1-9 \rho^{2}\right)\right\}\right]=\rho\left[1-\frac{1-\rho^{2}}{6}\left\{1-\frac{1}{12}\left(1-9 \rho^{2}\right)\right\}\right]$, 
and these we shall compare with the form

III.

$$
\bar{r}=\frac{2}{\pi}\left(\alpha+\cot \alpha-\alpha \cot ^{2} \alpha\right)
$$

\begin{tabular}{c|c|c|c|c|c|c|c|c|c|c}
$\rho$ & $\cdot 1000$ & $\cdot 2000$ & $\cdot 3000$ & 4000 & $\cdot 5000$ & $\cdot 6000$ & $\cdot 7000$ & $\cdot 8000$ & .9000 & 9500 \\
I & $\cdot 0853$ & $\cdot 1710$ & $\cdot 2578$ & $\cdot 3463$ & $\cdot 4377$ & 5333 & $\cdot 6347$ & $\cdot 7443$ & 8649 & .9304 \\
II & $\cdot 0847$ & $\cdot 1697$ & $\cdot 2555$ & $\cdot 3419$ & $\cdot 4310$ & $\cdot 5241$ & 6236 & $\cdot 7330$ & .8566 & 9254 \\
III & $\cdot 0850$ & $\cdot 1704$ & $\cdot 2570$ & $\cdot 3451$ & $\cdot 4360$ & 5301 & $\cdot 6290$ & $\cdot 7357$ & $\cdot 8540$ & 9209
\end{tabular}

It will be observed that the approximations lie on either side of the exact value over the greater part of the range, and that the error of the first approximation increases up to the value when $\rho=9$. The second formula gives the correct value somewhere between 8 and 9 , and is thereafter too large.

For the particular case

I find (formula III)

$\mathrm{Mr}$ Soper gives (p. 109) the value

and the experimental data

$$
\begin{aligned}
\rho= & \cdot 6608, \\
\bar{r}= & \cdot 5897, \text { nearly the maximum difference from } \rho, \\
& .5933
\end{aligned}
$$

.5609 .

The two theoretical values are much nearer to each other than either is to the experimental value. On the whole, it is obvious that even in this unfavourable case Mr Soper's formulae possess remarkable accuracy.

10. The use of the correlation coefficient $r$ as independent variable of these frequency curves is in some respects highly unsatisfactory. For high values of $r$ the curve becomes extremely distorted and cramped, and although this very cramping forces the mean $\bar{r}$ to approach $\rho$, the difference compared with $1-\rho$ becomes inordinately great. Even for high values of $n$, the distortion in this region becomes extreme, and since at the same time the curve rapidly changes its shape, the values of the mean and standard deviation cease to have any very useful meaning. It would appear essential in order to draw just conclusions from an observed high value of the correlation coefficient, say 99 , that the frequency curves should be reasonably constant in form.

The previous paragraphs suggest that more natural variables for the treatment of our formulae are afforded by the transformations

$$
\begin{aligned}
& t=\tan \beta=\frac{r}{\sqrt{1-r^{2}}}, \\
& \tau=\tan \alpha=\frac{\rho}{\sqrt{1}-\rho^{2}} .
\end{aligned}
$$

The expression for the frequency curve (II)

$$
\left(1-r^{2}\right)^{\frac{n-4}{2}}\left(\frac{\partial}{\sin \theta \partial \theta}\right)^{n-1} \frac{\theta^{2}}{2} d r
$$




\section{Distribution of the Correlation Coefficients of Samples}

now becomes

$$
\left(\frac{\partial}{\sin \theta \partial \theta}\right)^{n-1} \frac{\theta^{2}}{2} \frac{d t}{\left(1+t^{2}\right)^{\frac{n-1}{2}}}
$$

and the range of the curve is extended from $-\infty$ to $+\infty$.

It is interesting that in the important case, $r=0$, the frequency reduces to $\frac{d t}{\left(1+t^{2}\right)^{\frac{n-1}{2}}}$ and the curves are identical with those found by "Student" for $z$, the probability integral of which he has tabulated in his first paper.

11. The moments of these curves are obtained by the evaluation of the expressions

$$
\int_{-\infty}^{\infty}\left(\frac{\partial}{\sin \theta \partial \theta}\right)^{n-1} \frac{\theta^{2}}{2} \frac{d t}{\left(1+t^{2}\right)^{\frac{n-1}{2}}}, \int_{-\infty}^{\infty}\left(\frac{\partial}{\sin \theta \partial \theta}\right)^{n-1} \frac{\theta^{2}}{2} \frac{t d t}{\left(1+t^{2}\right)^{\frac{n-1}{2}}},
$$

and so on; of these the first is known already (III) to have the value

$$
\frac{\pi n-3}{\left(1-\rho^{2}\right)^{\frac{n-1}{2}}}
$$

and the others may be obtained in succession, for

$$
\begin{aligned}
I_{n, p} & =\int_{-\infty}^{\infty}(\sin \theta \partial \theta)^{n-1} \frac{\partial^{n-1}}{2} \cdots t^{p} d t{ }^{n-1}=\frac{\partial^{n-1}}{\partial \rho^{n-1}} \int_{-\infty}^{\infty} \frac{1}{r^{n-1}} \frac{\theta^{2}}{2} \frac{t^{p} d t}{\left(1+t^{2}\right)^{\frac{n-1}{2}}} \\
& =\frac{\partial^{n-1}}{\partial \rho^{n-1}} \int_{-\infty}^{\infty} \frac{\theta^{2}}{2} \cdot \frac{d t}{t^{n-1}-p}=\frac{\partial^{p}}{\partial \rho^{p}} I_{n-p, 0},
\end{aligned}
$$

so that the first moment

$$
\begin{gathered}
\int_{-\infty}^{\infty}\left(\begin{array}{c}
\partial \\
\sin \theta \partial \theta
\end{array}\right)^{n-1} \frac{\theta^{2}}{2} \cdot \frac{t d t}{\left(1+t^{2}\right)^{\frac{n-1}{2}}}=\frac{\partial}{\partial \rho} \cdot \frac{\pi p-4}{\left(1-\rho^{2}\right)^{\frac{n-2}{2}}}=\frac{\pi n-4(n-2) \rho}{\left(1-\rho^{2}\right)^{\frac{n-4}{2}}} \\
\bar{t}=\frac{n-2}{n-3} \frac{\rho}{\sqrt{1-\rho^{2}}}=\frac{n-2}{n-3} \tau
\end{gathered}
$$

hence

The mean, therefore, is greater than the true value $\tau$ by a constant fraction of its value. And this fraction decreases in the simplest possible manner as $n$ increases.

In the same way, we may evaluate the second moment,

$$
\bar{t}^{2}+\sigma^{2}=\frac{1}{n-4}\left\{1+(n-1) \tau^{2}\right\}
$$

and

the third moment

$$
\sigma^{2}=\frac{1}{n-4}\left\{1+\tau^{2}+\frac{(n-2)}{(n-3)^{2}} \tau^{2}\right\}
$$

$$
\sqrt{\beta_{1}} \sigma^{3}=\frac{(n-2) \tau}{(n-3)(n-4)(n-5)}\left\{3\left(1+\tau^{2}\right)+\frac{2 \tau^{2}(n-1)}{(n-3)^{2}}\right\},
$$

and the fourth moment 
$\beta_{2} \sigma^{4}=\frac{3}{(n-4)(n-6)}\left\{\left(1+\tau^{2}\right)^{2}+\frac{6(n-2) \tau^{2}}{(n-3)(n-5)}\left(1+\tau^{2}\right)+\frac{6(n-2)\left(3 n^{2}-11 n+12\right) \tau^{4}}{(n-3)^{4}(n-5)}\right\}$.

For high values of $n$, all but the first terms tend to vanish; $\beta_{1}$ tends to vary as $\rho^{2}$, and $\beta_{2}$ tends to become independent of $\rho$. In effect for high values of $r$, where $\rho^{2}$ is nearly equal to unity, the form of the curve is nearly constant, but the skewness measured by $\beta_{1}$ decreases to zero at the origin, and changes its sense, when $\tau$ and $\rho$ change their sign.

Tables are appended for inspection rather than for reference which show the nature and extent of these changes in the form of the curves.

Table of $\sigma^{2}$.

\begin{tabular}{|c|c|c|c|c|c|c|c|c|c|}
\hline$\tau^{2}=$ & $\cdot 01$ & .08 & $\cdot 10$ & .90 & 1.00 & $3 \cdot 00$ & $10 \cdot 00$ & $30 \cdot 10$ & $100 \cdot 00$ \\
\hline$n=$ & & & & & & & & & \\
\hline 8 & $\cdot 2531$ & $\cdot 2593$ & $\cdot 2810$ & $\cdot 3430$ & .5600 & $1 \cdot 140$ & $3 \cdot 350$ & $9 \cdot 550$ & $31 \cdot 250$ \\
\hline 18 & 1123 & $\cdot 1148$ & $\cdot 1234$ & 1481 & -2344 & $\cdot 4811$ & $1 \cdot 344$ & 3.811 & $12 \cdot 444$ \\
\hline 18 & .07219 & .07372 & .07908 & .09438 & $\cdot 1479$ & .3010 & 8365 & $2 \cdot 367$ & 7.722 \\
\hline 23 & .05319 & .05429 & $\cdot 05817$ & .06925 & $\cdot 1080$ & .2188 & .6066 & 1.714 & $5 \cdot 592$ \\
\hline 33 & .03484 & .03555 & $\cdot 03805$ & .04518 & 7015 & $\cdot 1415$ & $\cdot 3912$ & $1 \cdot 105$ & $3 \cdot 602$ \\
\hline 43 & $\cdot 02590$ & .02643 & .02827 & .03353 & .05194 & $\cdot 1045$ & $\cdot 2886$ & $\cdot 8146$ & $2 \cdot 65 \tilde{j}$ \\
\hline 58 & 02062 & .02103 & .02249 & $\cdot 02666$ & .04123 & 08288 & 2287 & 6451 & $2 \cdot 103$ \\
\hline
\end{tabular}

Table of $\beta_{1}$.

\begin{tabular}{|c|c|c|c|c|c|c|c|c|c|c|}
\hline$\tau^{2}=$ & $\cdot 01$ & .03 & $\cdot 10$ & .80 & 1.00 & $3 \cdot 00$ & $10 \cdot 00$ & $30 \cdot 10$ & $100) \cdot(00)$ & $\propto$ \\
\hline \multicolumn{11}{|l|}{$n=$} \\
\hline 8 & $\cdot 05685$ & $\cdot 1662$ & 5076 & $1 \cdot 230$ & $2 \cdot 450$ & $3 \cdot 788$ & 3.965 & $4 \cdot 153$ & $4 \cdot 184$ & $4 \cdot 252$ \\
\hline 18 & 01517 & $\cdot 04776$ & $\cdot 1376$ & $\cdot 3400$ & 7058 & 1.018 & $1 \cdot 205$ & 1271 & $1 \cdot 296$ & 1.3065 \\
\hline 18 & .008399 & 02463 & 07645 & $\cdot 1914$ & $\cdot 4016$ & 5857 & 6990 & 7395 & 7546 & $\cdot 7619$ \\
\hline 23 & $\cdot 005757$ & .01691 & 05247 & $\cdot 1317$ & $\cdot 3016$ & $\cdot 4093$ & $\cdot 4910$ & 5208 & $\cdot 5314$ & 5.361 \\
\hline 39 & $\cdot 003518$ & $\cdot 01035$ & 03214 & 08100 & $\cdot 1731$ & 2559 & 3031 & 3260 & -33334 & 3366 \\
\hline 43 & $\cdot 002530$ & 007435 & 02315 & 05841 & $\cdot 125 \mathrm{I}$ & $\cdot 1858$ & $\cdot 2237$ & $\cdot 2376$ & 2429 & $\cdot 2452$ \\
\hline 53 & $\cdot 001973$ & .005798 & 01807 & $\cdot 04562$ & $\cdot 09800$ & $\cdot 1458$ & $\cdot 1757$ & $\cdot 1868$ & $\cdot 1910$ & $\cdot 1928$ \\
\hline
\end{tabular}

Table of $\beta_{2}$.

\begin{tabular}{|c|c|c|c|c|c|c|c|c|c|c|c|}
\hline$T^{2}=$ & 00 & .01 & .09 & $\cdot 10$ & $\cdot 30$ & 1.00 & 8.00 & $10 \cdot 00$ & $30 \cdot 00$ & $100 \cdot 00$ & $\infty$ \\
\hline$n=$ & & & & & & & & & & & \\
\hline 8 & 6.0000 & 6.1137 & 6.3179 & 70179 & $8 \cdot 4767$ & $10 \cdot 9668$ & $12 \cdot 9652$ & $14 \cdot 1116$ & 14.5024 & $14 \cdot 6508$ & $14 \cdot 7159$ \\
\hline 13 & 3.8571 & $3 \cdot 8802$ & $3 \cdot 9248$ & 4.0663 & $4 \cdot 3770$ & $4 \cdot 9397$ & $5 \cdot 4240$ & 5.7147 & $5 \cdot 8186$ & 5.8578 & $5 \cdot 8750$ \\
\hline 18 & 3.5000 & $3.512 \mathrm{I}$ & $3 \cdot 5356$ & $3 \cdot 6104$ & $3 \cdot 7937$ & $4 \cdot 0828$ & $4 \cdot 35.32$ & $4 \cdot 5186$ & 4.5783 & 46009 & $4 \cdot 6109$ \\
\hline 23 & $3 \cdot 3529$ & $3 \cdot 3612$ & $3 \cdot 3768$ & 3.4271 & 3.5556 & $3 \cdot 7486$ & 3.9356 & 4.0511 & 4.0930 & $4 \cdot 1089$ & $4 \cdot 1159$ \\
\hline 38 & $3 \cdot 2222$ & $3 \cdot 2271$ & $3 \cdot 2365$ & $3 \cdot 2667$ & $3 \cdot 3343$ & $3 \cdot 4619$ & $3 \cdot 5773$ & $3 \cdot 6493$ & $3 \cdot 6756$ & $3 \cdot 6856$ & $3 \cdot 6899$ \\
\hline 43 & $3 \cdot 1622$ & $3 \cdot 1656$ & $3 \cdot 1723$ & $3 \cdot 1938$ & $3 \cdot 2422$ & $3 \cdot 3261$ & $3 \cdot 4172$ & $3 \cdot 4692$ & $3 \cdot 4886$ & $3 \cdot 4958$ & $3 \cdot 4991$ \\
\hline 58 & $3 \cdot 1277$ & $3 \cdot 1303$ & $3 \cdot 1356$ & $3 \cdot 1522$ & $3 \cdot 1898$ & $3 \cdot 2640$ & $3 \cdot 3281$ & $3 \cdot 3676$ & $3 \cdot 3826$ & $3 \cdot 3883$ & $3 \cdot 3909$ \\
\hline
\end{tabular}


12. The fact that the mean value $\vec{r}$ of the observed correlation coefficient is numerically less than $\rho$ might have been interpreted as meaning that given a single observed value $r$, the true value of the correlation coefficient of the population from which the sample is drawn is likely to be greater than $r$. This reasoning is altogether fallacious. The mean $\bar{r}$ is not an intrinsic feature of the frequency distribution. It depends upon the choice of the particular variable $r$ in terms of which the frequency distribution is represented. When we use $t$ as variable, the situation is reversed. Whereas in using $r$ we cramp all the high values of the correlation into the small space in the neighbourhood of $r=1$, producing a frequency curve which trails out in the negative direction and so tending to reduce the value of the mean, by using $t$, we spread out the region of high values, producing asymmetry in the opposite sense, and obtain a value $\bar{t}$ which is greater than $\tau$. The mean might, in fact, be brought to any chosen point, by stretching and compressing different parts of the scale in the required manner. For the interpretation of a single observation the relation between $\bar{t}$ and $\tau$ is in no way superior to that between $\bar{r}$ and $\rho$. The variable $t$ has been chosen primarily in order to give stability of form to the frequency curves in different parts of the scale. It is in addition a variable to which the analysis naturally leads us, and which enables the mean and moments to be readily calculated, and so a comparison to be made with the standard Pearson curves, but it is not, with these advantages, in a unique position. In some respects the function, $\log \tan \frac{1}{2}\left(\alpha+\frac{\pi}{2}\right)$, is its superior as independent variable.

I have given elsewhere* a criterion, independent of scaling, suitable for obtaining the relation between an ubserved correlation of a sample and the mest. probable value of the correlation of the whole population. Since the chance of any observation falling in the range $d \cdot$ is proportional to

$$
\left(1-\rho^{2}\right)^{2-1}(1-, \cdots)^{2+4}(\sin \theta \partial \theta)^{n-1} \frac{\theta^{2}}{2} d r
$$

for variations of $\rho$, we must find that value of $\rho$ for which this quantity is a maximum, and thereby ubtain the equation

$$
\frac{\partial}{\partial \rho}\left\{\left(1-\rho^{*}\right)^{n-1}(\sin \theta \partial \dot{\theta})^{n-1} \frac{\theta^{2}}{2}\right\}=0 .
$$

Since

$$
\int_{0}^{x} \frac{d x}{(\cosh x+\cos \theta)^{n-1}}=\frac{1}{1 n-1}\left(\begin{array}{c}
\partial \\
\sin \theta \partial \theta
\end{array}\right)^{n-1} \frac{\theta^{2}}{2}
$$

we have $\quad \int_{0}^{x} \partial \rho\left\{\left(1-\rho^{2}\right)^{n-1} \frac{d x}{(\cosh x+\cos \theta)^{n-1}}\right\}=0$,

* R. A. Fisher, "On an absolute criterion for fitting frequency curves," Messenger of Mathematics, Feluruary, 1912. 
which leads by a process of simplification to the equation

$$
\int_{0}^{\infty} \frac{d x}{(\cosh x-\rho r)^{n}}(r-\rho \cosh x)=0 .
$$

Since $\cosh x$ is always greater than $\rho r$, the factor in the numerator, $r-\rho \cosh x$, must change sign in the range of integration. We therefore see that $r$ is greater than $\rho$. Further an approximate solution may be obtained for large values of $n$. The integrand is negligible save when $x$ is very small, and we may write

$$
1+\frac{x^{2}}{2} \text { for } \cosh x
$$

and

$$
(1-\rho r)^{n} e^{\frac{n x^{2}}{2(1-\rho r)}} \text { for }(\cosh x-\rho r)^{n} \text {. }
$$

Then $\quad r \int_{0}^{\infty} e^{-\frac{n x^{2}}{2(1-\rho r)}} d x=\rho \int_{0}^{\infty}\left(1+\frac{x^{2}}{2}\right) e^{-\frac{n x^{2}}{2(1-\rho r)}} d x$, and in consequence, as a first approximation,

$$
r=\rho\left(1+\frac{1-r^{2}}{2 n}\right)
$$

'The corresponding relation between $t$ and $\tau$ is evidently

$$
t=\tau\left(1+\frac{1}{2 n}\right)
$$

It is now apparent that the most likely value of the correlation will in general be less than that observed, but the difference will be only half of that suggested by the mean, $\bar{t}$.

It might plausibly be urged that in the choice of an independent variable we should aim at making the relation between the mean and the true values approach the above equation, or rather that to which the above is an approximation, or that we should aim at reducing the asymmetry of the curves, or at approximate constancy of the standard deviation. In these respects the function

$$
\log \tan \frac{1}{2}\left(\alpha+\frac{\pi}{2}\right) \text { that is, } \tanh ^{-1} \rho
$$

is not a little attractive, but so far as $I$ have examined it, it does not tend to simplify the analysis, and approaches relative constancy at the expense of the constancy proportionate to the variable, which the expressions in $\tau$ exhibit . 